\title{
Analysis and modelling of ATM (AAL5) traffic traces
}

\author{
Madhu D. K. Bhabuta and Peter G. Harrison *† \\ Imperial College of Science, Technology and Medicine \\ Department of Computing \\ 180 Queen's Gate, London SW7 2BZ
}

\begin{abstract}
We present an analysis of Asynchronous Transfer Mode (ATM) traffic data collected from the London MAN (Metropolitan Area Network). The traffic is found to have different characteristics depending on its intensity. The correlations between inter-arrival times of sessions for traffic from heavily utilised sites is insignificant at the $5 \%$ significance level and a Poisson Point process is thus appropriate as a model. Traffic from low utilisation sites, on the other hand, shows long-memory characteristics with correlation at lag 40 and more. This traffic is modelled firstly using an autoregressive process and then the well known Markov Modulated Poisson Process (MMPP). The burst size distribution for both correlated and uncorrelated traffic shows distinct peaks which corresponds to the fragmentation as data travels through networks with different Maximum Transport Units (MTUs).
\end{abstract}

\section{Introduction}

ATM technology is being standardised as the networking technology for many of the emerging wide area Broadband Integrated Service Digital Networks (BISDN) protocol stacks. It is thus important to have a good understanding of the behaviour of the traffic and its load so that any analytical models that are built to predict performance take a realistic workload model as input. Unrealistic workload models can easily lead to either very pessimistic or optimistic predictions [12].

Some work that has been done on Ethernet traces e.g. [15], [20] and [26] has cast doubt on the use of Poisson arrival processes as models of the traffic. We have set out to study whether this too is the case for ATM networks and how we

${ }^{*}$ The authors would like to thank Jonathan Couzens of the London MAN for his help in the monitoring and capture of data used in this research.

${ }^{\dagger}$ mb3, pgh@ic.ac.uk

The original version of this chapter was revised: The copyright line was incorrect. This has been corrected. The Erratum to this chapter is available at DOI: 10.1007/978-0-387-35353-1_28

D. Kouvatsos (ed.), Performance Analysis of ATM Networks

(C) IFIP International Federation for Information Processing 2000 
may model the traffic. We have collected and analysed ATM traffic at cell level and present models for it based on traffic and the workings of network protocols through which the traffic travels. The paper starts with a description of the experimental setup and the capture procedure. In section 2, the traffic data is analysed and the two main categories that the traces fall into are identified. In section 3 we present an analysis of the traffic data based on methods employed in time series analysis and show how traffic from sites that have heavy traffic differs from light traffic. Section 4 considers models for correlated traffic using techniques from time series analysis. The autocorrelation function is then used to parameterise a Markov Modulated Poisson Process (MMPP). In section 5 we consider the burst-size distribution of cells and present models for it. The paper concludes in Section 6.

\section{Measuring IP traffic over ATM}

There are very few ATM networks that are sufficiently utilised to provide representative traces. However, the network from which our measurements are taken is the London MAN which has high utilisation due to Telehouse which is the hub for the UK's commercial Internet traffic and is one of the nodes on the network. The data obtained from this network is live and not under controlled experimental conditions.

Figure 1 illustrates the network configuration for the MAN and an enlarged view of the Imperial College node where traffic was captured. The switch is a Fore Systems' ASX-200 ATM switch; the connection with the MAN is a 155 $\mathrm{Mbs}^{-1}$ OC-3c multi-mode fibre link. The router is a Cisco router with a Cisco API ATM adapter card interfacing with the switch. The adapter layer embeds a dedicated processor and special-purpose hardware to handle AAL5. The physical layers of the LANs in the sites on the London MAN consist entirely of Ethernet.

\subsection{Measurement Tool}

The data is collected using a Hewlett Packard Broadband Series Test System at the point indicated on Figure 1. Incoming data is captured at full line rates up to $155 \mathrm{Mb} / \mathrm{s}$. The switch is configured so as to make a copy of each cell and send it to a spare port to which the analyser is attached.

The analyser has 8Mbytes of RAM, enough to capture 131,072 ATM cells (including resassembly overhead) in one session. The system can capture in both continuous (write to buffer in circular fashion) and burst mode (stop writing to the buffer when it is full). We used the burst mode mainly and were able to capture cells for upto 30 minutes for sites with low-utilisation and up to 1 minute on sites with heavy utilisation. All inter-arrival times can be measured to an accuracy of 10 nanoseconds on this system. The captured cells are illustrated in Figure 2. The low-order bit of the payload type field of the ATM cell is used 


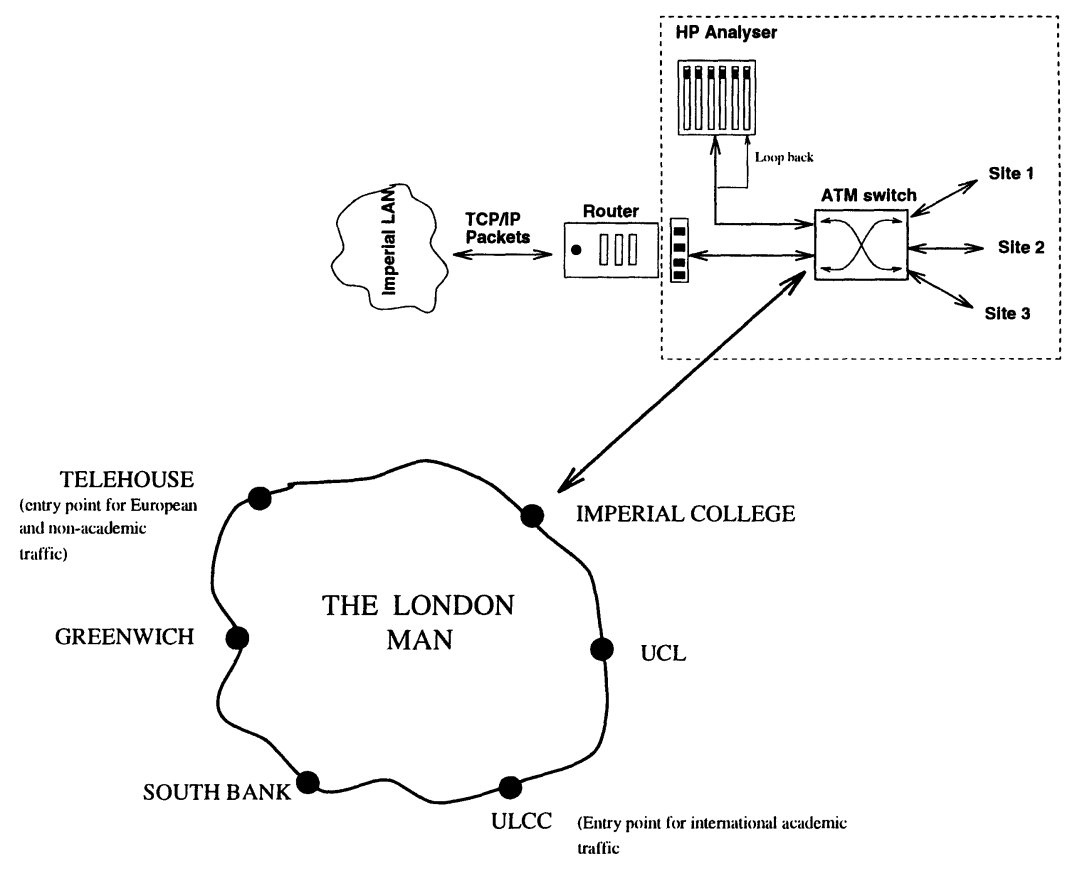

Figure 1: The Experimental Site

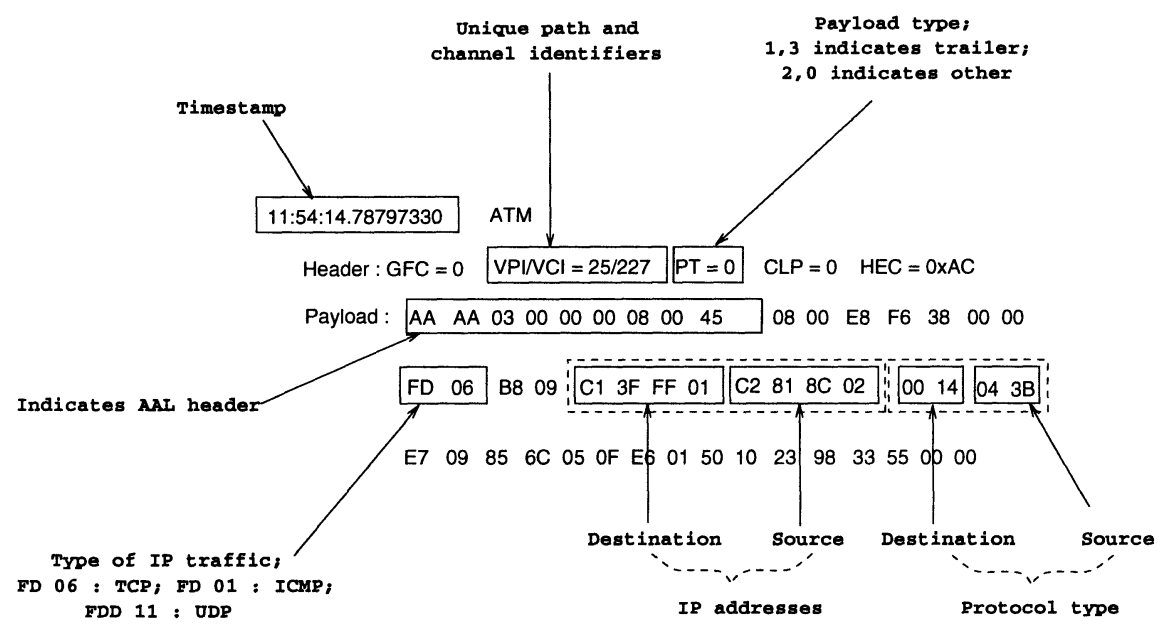

Figure 2: An example captured header cell 
to mark the end of the final cell in a packet. If this bit is 1 or 3 the cell is the last in the packet. 2 or 0 is used to indicate congestion and 4, 5, 6 or 7 is used for resource reservation.

\subsection{Experimental Procedure}

We have conducted many captures per switch port, each data collection having been done at different times and on different days so as to limit the effect of statistical variations. Table 1 gives the times at which each capture started and ended to the nearest second. The intensity column of the table gives the levels of intensity $\mathrm{X} / \mathrm{Y}$ where $\mathrm{X}$ is the intensity when the capture was started and $\mathrm{Y}$ is the intensity when the capture stopped. There are 3 intensity levels; $\operatorname{Low}(\mathrm{L}), \operatorname{High}(\mathrm{H})$ and Moderate(M). The data was captured on weekdays only as we noticed a marked decrease in usage patterns on Saturday and Sunday. By so doing, we have also eliminated a potential source of seasonality in our data.

The data is fed through a series of filters which classify each cell according to the IP protocol: TCP, UDP or ICMP. The filters are written in Perl 5 and based on pattern matching. Perl was chosen as the language to write the filters because it is optimised for pattern matching and it is easy to pipe data in parallel to as many files as desired.

We match on the different parts of a cell and pipe the desired statistics e.g. the time-stamp to an appropriate file. First the AAL header is matched with the standard IP header to identify the start of an IP packet, then the IP protocol is identified and finally pattern matching is done on the type of protocol (see Fig. 2). The largest proportion of traffic was TCP(80\%), UDP accounted for $10 \%$ and ICMP was less than $0.01 \%$ of the traffic. The main constituents of the TCP traffic were FTP, Telnet, SMTP and Web (or HTTP). As the proportion of traffic from ICMP, other TCP and UDP was so small, it was collectively classified under "miscellaneous".

The inter-arrival times between cells are obtained in the usual way, by taking the difference between timestamps of successive cells.

The filtered data clearly showed :-

- The inter-arrival time between cells that belonged to the same packet is of the order of $8 \mu \mathrm{s}-20 \mu \mathrm{s}$ and $10 \mathrm{~ms}-1 \mathrm{~s}$ between successive packets.

- The minimum number of cells within a packet is 2 and the maximum is 32.

This indicates that because inter-arrival times of packets $\gg$ inter-arrival times of cells within a packet, we must analyse the two separately. Hence we analyse our data as arrival points of batches and define a batch as an arrival composed of 2 or more cells. Our analysis concentrates on analysis and modelling of inter-arrival times between cells and the burst-size distribution at each arrival instant. 


\begin{tabular}{|c|l|l|l|l|c|}
\hline S.no & Date & Start & End & Data set & Intensity \\
\hline 1 & $04 / 02 / 97$ & $11: 54: 14$ & $11: 54: 35$ & $\mathrm{t} 9702041154$ & $\mathrm{H} / \mathrm{H}$ \\
2 & $04 / 02 / 97$ & $14: 20: 13$ & $14: 20: 15$ & $\mathrm{t} 9702041420$ & $\mathrm{H} / \mathrm{H}$ \\
3 & $06 / 02 / 97$ & $12: 33: 43$ & $13: 03: 25$ & $\mathrm{t} 9702061233$ & $\mathrm{H} / \mathrm{M}$ \\
4 & $06 / 02 / 97$ & $14: 39: 46$ & $15: 50: 05$ & $\mathrm{t} 9702061439$ & $\mathrm{H} / \mathrm{H}$ \\
5 & $06 / 02 / 97$ & $18: 23: 59$ & $18: 24: 21$ & $\mathrm{t} 9702061823$ & $\mathrm{M} / \mathrm{M}$ \\
6 & $06 / 02 / 97$ & $19: 31: 57$ & $19: 32: 09$ & $\mathrm{t} 9702061931$ & $\mathrm{~L} / \mathrm{L}$ \\
7 & $07 / 02 / 97$ & $14: 50: 39$ & $14: 50: 55$ & $\mathrm{t} 9702071450$ & $\mathrm{H} / \mathrm{H}$ \\
8 & $07 / 02 / 97$ & $15: 55: 10$ & $15: 55: 12$ & $\mathrm{t} 9702071555$ & $\mathrm{H} / \mathrm{H}$ \\
9 & $07 / 02 / 97$ & $17: 06: 53$ & $17: 07: 10$ & $\mathrm{t} 9702071706$ & $\mathrm{M} / \mathrm{M}$ \\
10 & $10 / 02 / 97$ & $11: 51: 04$ & $11: 51: 23$ & $\mathrm{t} 9702101151$ & $\mathrm{H} / \mathrm{H}$ \\
11 & $10 / 02 / 97$ & $16: 59: 32$ & $16: 59: 45$ & $\mathrm{t} 9702101659$ & $\mathrm{H} / \mathrm{H}$ \\
12 & $11 / 02 / 97$ & $07: 18: 59$ & $07: 19: 35$ & $\mathrm{t} 9702110718$ & $\mathrm{~L} / \mathrm{L}$ \\
13 & $11 / 02 / 97$ & $14: 55: 24$ & $14: 55: 32$ & $\mathrm{t} 9702111455$ & $\mathrm{H} / \mathrm{H}$ \\
14 & $12 / 02 / 97$ & $09: 47: 13$ & $09: 47: 37$ & $\mathrm{t} 9702120947$ & $\mathrm{~L} / \mathrm{L}$ \\
15 & $12 / 02 / 97$ & $14: 53: 54$ & $14: 54: 03$ & $\mathrm{t} 9702121453$ & $\mathrm{M} / \mathrm{M}$ \\
16 & $13 / 02 / 97$ & $10: 09: 09$ & $10: 09: 39$ & $\mathrm{t} 9702131009$ & $\mathrm{H} / \mathrm{H}$ \\
17 & $18 / 02 / 97$ & $11: 27: 03$ & $11: 33: 48$ & $\mathrm{t} 9703181127$ & $\mathrm{H} / \mathrm{H}$ \\
18 & $18 / 02 / 97$ & $16: 12: 32$ & $16: 18: 50$ & $\mathrm{t} 9703181612$ & $\mathrm{H} / \mathrm{H}$ \\
19 & $18 / 02 / 97$ & $18: 20: 52$ & $18: 28: 02$ & $\mathrm{t} 9703181820$ & $\mathrm{M} / \mathrm{M}$ \\
20 & $19 / 03 / 97$ & $13: 08: 05$ & $13: 14: 55$ & $\mathrm{t} 9703191308$ & $\mathrm{H} / \mathrm{H}$ \\
21 & $24 / 03 / 97$ & $10: 16: 49$ & $10: 17: 10$ & $\mathrm{t} 9703241016$ & $\mathrm{H} / \mathrm{H}$ \\
22 & $24 / 03 / 97$ & $13: 45: 26$ & $13: 45: 38$ & $\mathrm{t} 9703241345$ & $\mathrm{H} / \mathrm{H}$ \\
23 & $24 / 03 / 97$ & $14: 51: 17$ & $14: 51: 31$ & $\mathrm{t} 9703241451$ & $\mathrm{M} / \mathrm{M}$ \\
24 & $24 / 03 / 97$ & $15: 58: 39$ & $15: 58: 49$ & $\mathrm{t} 9703241558$ & $\mathrm{H} / \mathrm{H}$ \\
25 & $02 / 04 / 97$ & $14: 54: 07$ & $14: 54: 21$ & $\mathrm{t} 9704021454$ & $\mathrm{~L} / \mathrm{H}$ \\
26 & $02 / 04 / 97$ & $15: 56: 28$ & $15: 56: 46$ & $\mathrm{t} 9704021556$ & $\mathrm{H} / \mathrm{H}$ \\
27 & $02 / 04 / 97$ & $17: 19: 27$ & $17: 19: 44$ & $\mathrm{t} 9704021719$ & $\mathrm{H} / \mathrm{H}$ \\
28 & $02 / 04 / 97$ & $18: 32: 51$ & $18: 33: 07$ & $\mathrm{t} 9704021832$ & $\mathrm{M} / \mathrm{M}$ \\
29 & $03 / 04 / 97$ & $08: 33: 42$ & $08: 34: 06$ & $\mathrm{t} 9704030832$ & $\mathrm{~L} / \mathrm{L}$ \\
30 & $03 / 04 / 97$ & $11: 08: 19$ & $11: 08: 38$ & $\mathrm{t} 9704031108$ & $\mathrm{H} / \mathrm{M}$ \\
31 & $03 / 04 / 97$ & $12: 14: 25$ & $12: 14: 44$ & $\mathrm{t} 9704031214$ & $\mathrm{H} / \mathrm{H}$ \\
32 & $03 / 04 / 97$ & $14: 12: 55$ & $15: 24: 40$ & $\mathrm{t} 9704031412$ & $\mathrm{H} / \mathrm{M}$ \\
33 & $03 / 04 / 97$ & $16: 09: 37$ & $19: 00: 09$ & $\mathrm{t} 9704031609$ & $\mathrm{H} / \mathrm{N}$ \\
34 & $04 / 04 / 97$ & $12: 56: 29$ & $14: 27: 05$ & $\mathrm{t} 9704041256$ & $\mathrm{M} / \mathrm{H}$ \\
\hline
\end{tabular}

Table 1: Traffic log

\section{Analysis of inter-arrival times}

We feed the data through a filter that generates inter-arrival times of batches and perform identical data analysis on each of the sets in Table 1. The results can be divided into 2 categories; those for heavy traffic channels and those for light traffic channels. We have focused on 2 sets: t9702041154, representative of heavy traffic, and t9702061439, representative of light traffic. A full set of results from each data set in Table 1 can be found in [2]

To test for correlation, we compute the auto-correlation function of the interarrival times given by equation 1 for lag $k=1 \ldots N$ where $\mathrm{N}$ is the number of data points in the traffic log.

The auto-correlation coefficient $\rho_{k}$ at lag $\mathrm{k}$ is given by :- 


$$
\rho_{k}=\frac{\sum_{t=1}^{N-k}\left(x_{t}-\bar{x}\right)\left(x_{t+k}-\bar{x}\right)}{\sum_{t=1}^{N}\left(x_{t}-\bar{x}\right)^{2}}
$$

where $x_{t}$ is the inter-arrival time at time $t$ and $\bar{x}$ is the mean inter-arrival time.

In the first instance we plot correlograms (a plot in which $\rho_{k}$ is plotted against $k$ ) for each protocol type and data set, Figures 3, 4, 7 and 8 . The approximate $95 \%$ confidence limits at $\pm 2 / \sqrt{ } N$ are used to test the null hypothesis that the data is random. We can assume that the time series are stationary because :-

- The correlograms decay to zero reasonably quickly.

- From our analysis of the 34 data sets in Table 1, we noticed that the correlograms of data sets with the same intensity, have the same characteristics regardless of the day or time the data was collected. We thus proceed showing typical traffic behaviour from the sites with heavy utilisation and that from sites with low utilisation.

\subsection{Typical correlograms from sites with heavy utilisation}

Plots from high utilisation sites (Figures 3 and 4), show that less than $5 \%$ of the values of $\rho_{k}$ lie outside the $95 \%$ confidence limits and those that do, are at apparently arbitrary lags. We can thus conclude (see [3], [4]) that there is no firm evidence to reject the hypothesis that the observations are independently distributed.

Inorder to check whether the inter-arrival times of batches of cells can be modelled with an exponential distribution, the data is binned into histograms and the exponential function

$$
f(x)=\alpha e^{-\lambda x}
$$

fitted using the least squares method. The results for aggregate traffic from data set t9702041154 are shown in Figure 5. Figure 5A shows the fitting of an exponential function to the histogram of inter-arrival data by taking the original definition of a batch as in section 2.2, Fig. 5B shows a fit on considering cells arriving within 0-0.0001s as being part of a batch and Fig. $5 \mathrm{C}$ shows the best fit when cells arriving within 0-0.0002s are considered as part of a batch. The plots suggest that the tail of the distribution in Fig. 5A is exponential. We postulate that reason the first bins show divergence from exponential behaviour is due to a second level of burstiness at the IP (or packet) level.

In order to investigate this, we pass the data through another filter that uses a second definition of a batch. This time we consider all adjacent transmissions with the same source-destination pair (see Fig. 2) as a batch arrival. When we now plot the histogram of inter-arrival times and do a non-linear least squares 

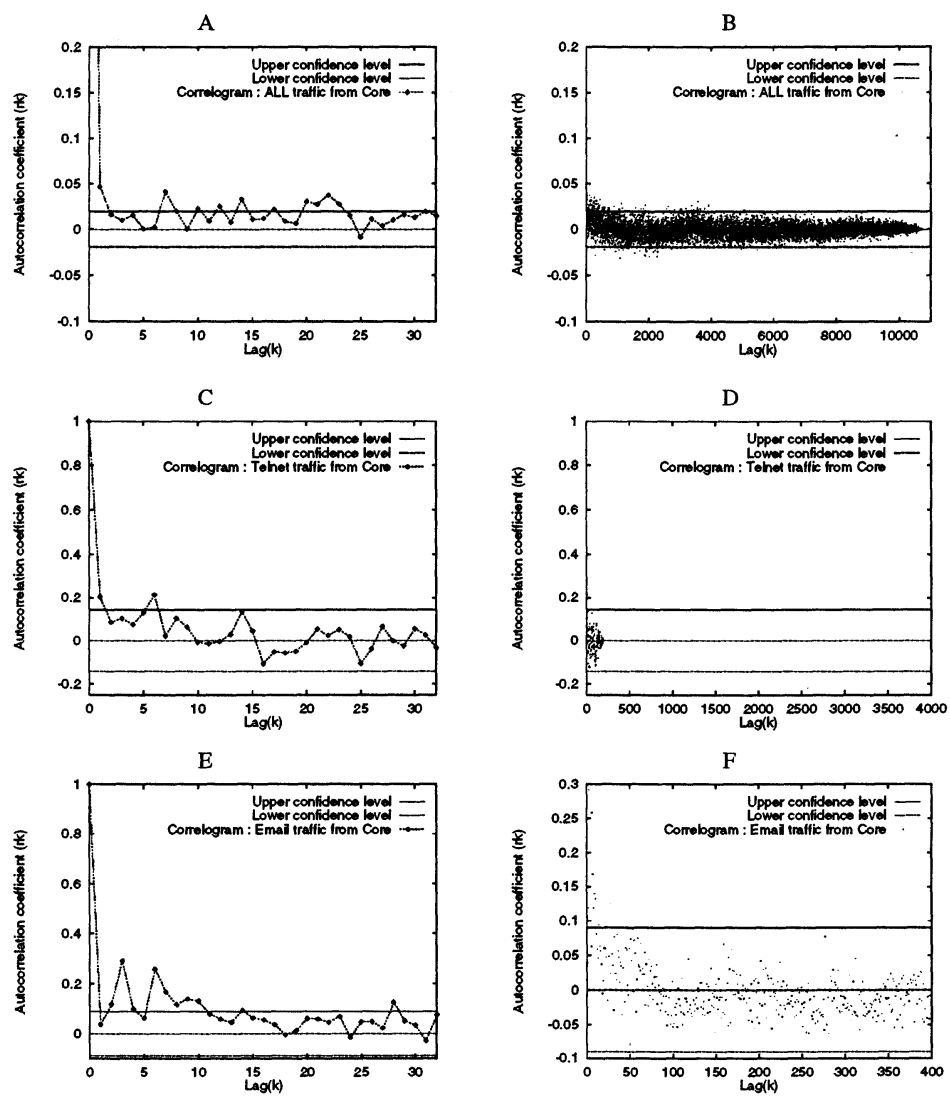

Figure 3: Autocorrelation function of inter-packet times. Ref : T9702041154 

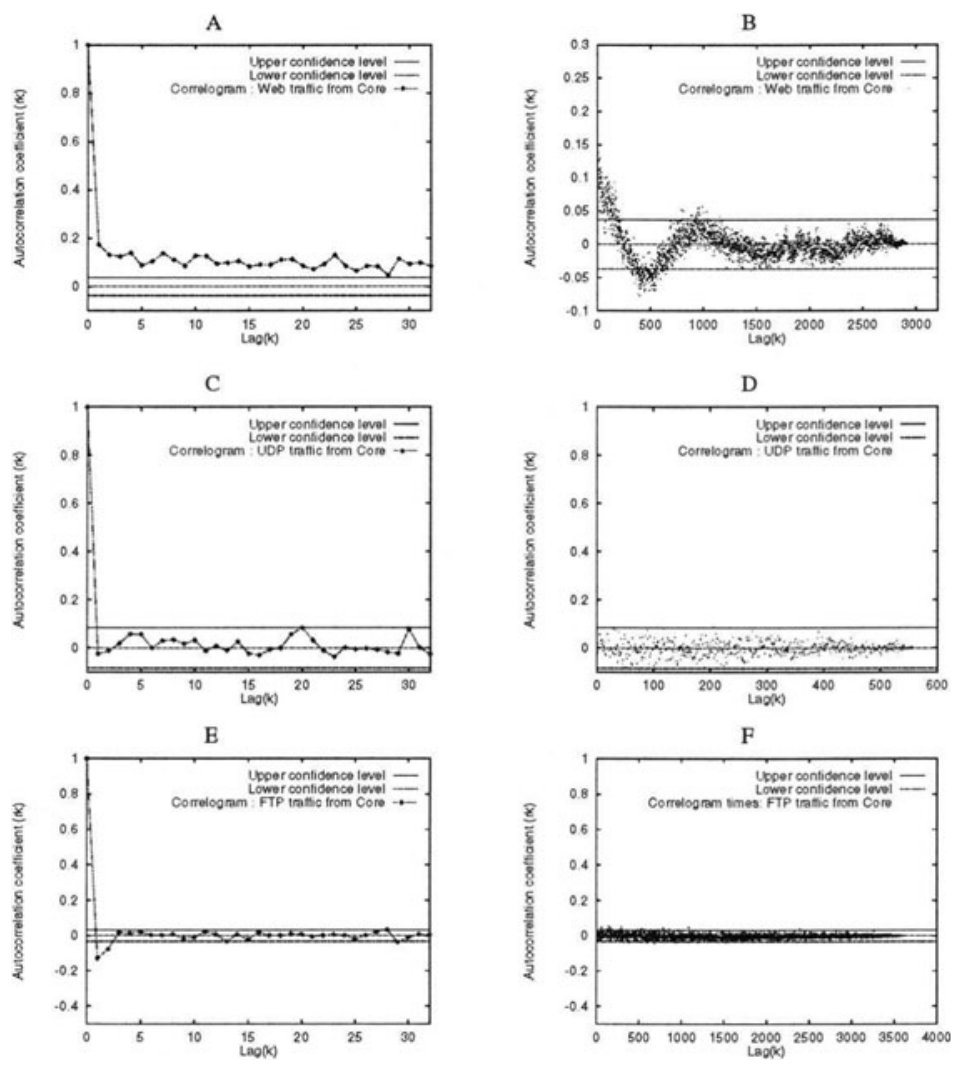

Figure 4: Autocorrelation function of inter-packet times. Ref : T9702041154

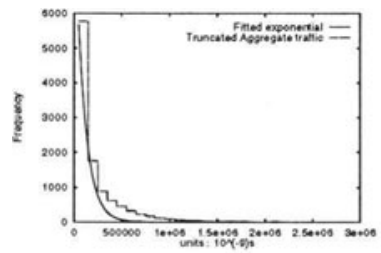

A

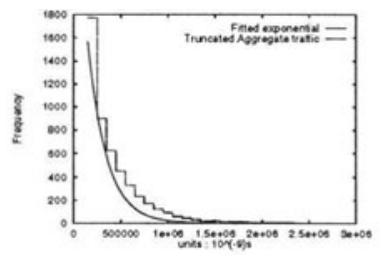

B

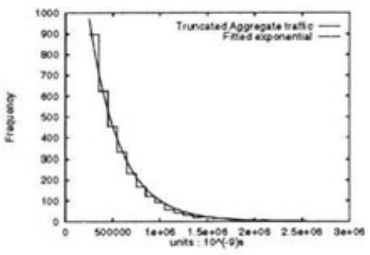

c

Figure 5: Fitting aggregate traffic data from set T9702041154 


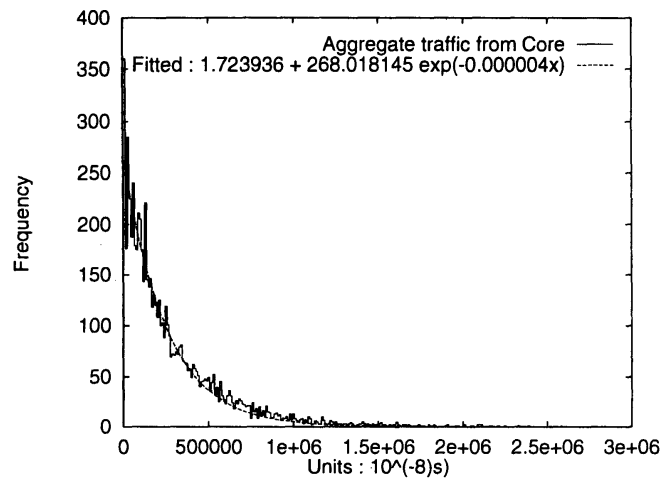

Figure 6: TCP batches removed from inter-arrival times set : T9702041154

fit, we find that we have a good fit (Figure 6). This indicates that the interarrival times between TCP/IP sessions are exponential and that a Poisson point process is a good model for these arrivals. To test the goodness of fit, we perform the $\chi^{2}$ test. The number of degrees of freedom $(\nu)$ is 258 . The $\chi^{2}$ statistic is 302.09677 ; for $\nu>100, \sqrt{2 \chi^{2}}$ is approximately normally distributed with mean $\sqrt{2 \nu-1}$ and unit variance. Thus the critical value $\chi_{0.05,258}=303.9003$. Since $302.09677<303.9003$, we do not reject the hypothesis that an exponential distribution is an appropriate model.

\subsection{Typical correlograms from sites with light utilisation}

Correlograms from the low utilisation sites (Figures 7 and 8) show different behaviour. Inter-burst arrival times for traces of aggregate traffic and the web protocol from set Tt9702061439 display heavy correlation at long lags, indicating long memory behaviour. From our analysis of data sets with L/L intensity (table 1), we find that the correlogram of inter-burst times of aggregate traffic always displays long-memory characteristics. The major cause of this behaviour are the long sessions in Web traffic, as evident in Figure 7. In fact, up to a lag of 80 , there is very little difference in correlogram from Web traffic and that from the aggregate traffic.

We propose to model the long-memory behaviour using time-series models. The choice of which type of process to fit is notoriously difficult [3], [13]. However, recent studies in [24] show that Autoregressive (AR) models can be used effectively as models for data with long-memory or long-range dependence and are in fact almost as effective as the powerful FARMA models which are a generalisation of autoregressive integrated moving average models in which the degree of differencing is allowed to take fractional values. Fitting AR processes to the data entails finding out the order and parameters of the process.

However, AR models can only be used to simulate a time series. In order 

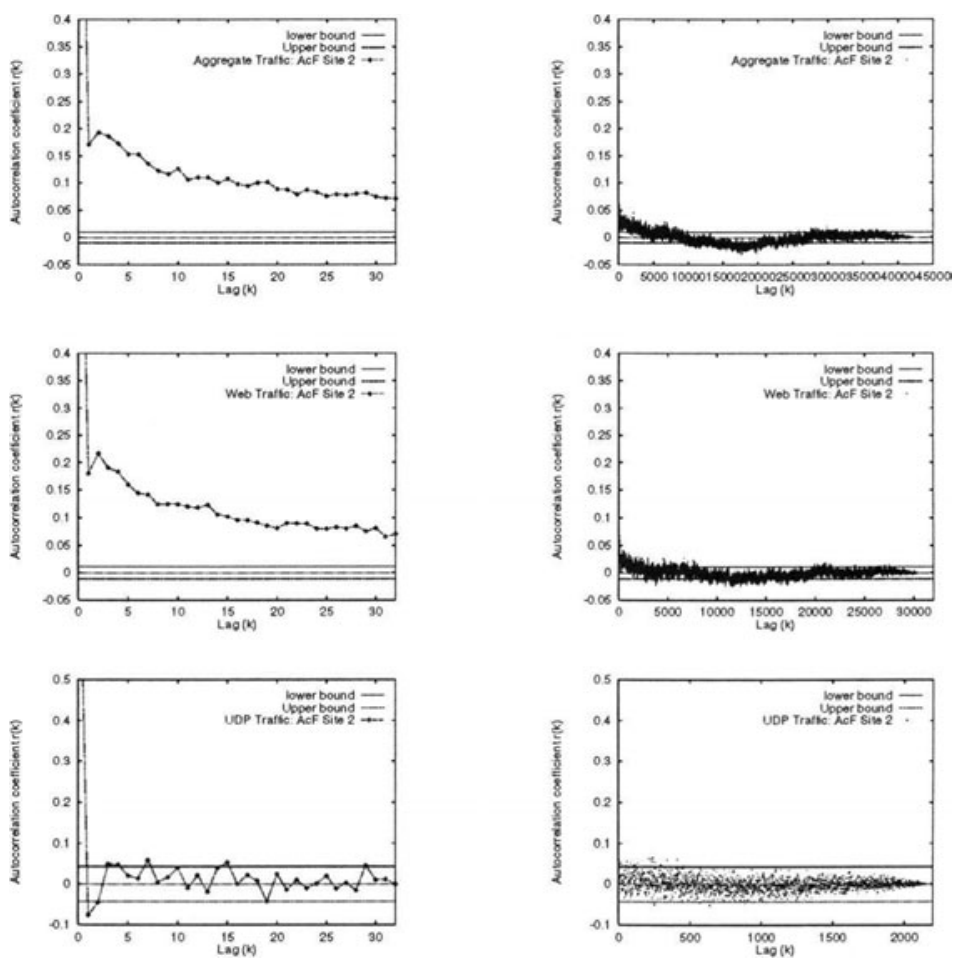

Figure 7: Autocorrelation function of inter-packet times. Ref : Tt9702061439 

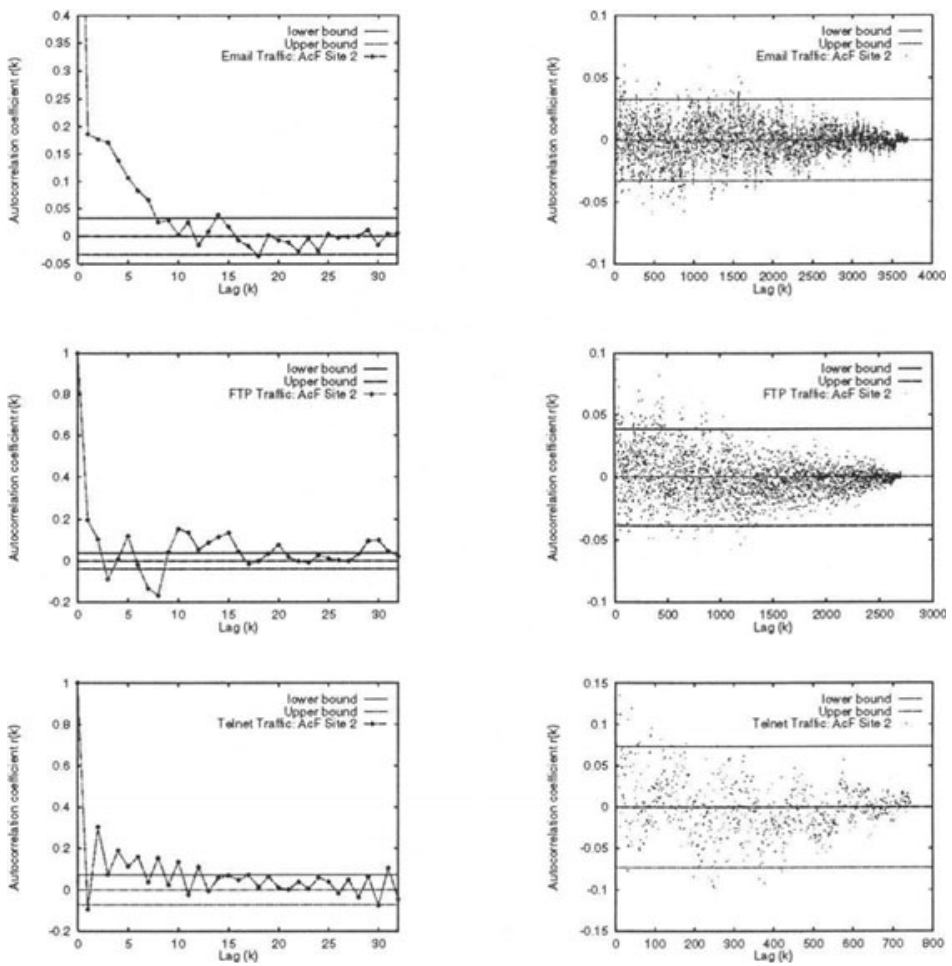

Figure 8: Autocorrelation function of inter-packet times. Ref : Tt9702061439 
to create work load models for queueing network-type models, we aim to show how a stochastic process, the well-known MMPP process (section 4) that can capture the correlation structure of the inter-arrival times. We also show how the MMPP can be parameterised using just the auto-correlation function.

\section{Models for correlated traffic}

\subsection{A time series model}

An autoregressive process $\left\{X_{t}\right\}$ of order $p$, with mean $\mu$, is given by

$$
X_{t}-\mu=\alpha_{1}\left(X_{t-1}-\mu\right)+\ldots+\alpha_{p}\left(X_{t-p}-\mu\right)+Z_{t}
$$

where $Z_{t}$ is a Normal random variable with zero mean. The parameters $\mu$ and $\alpha_{1}, \ldots, \alpha_{p}$ may be estimated by substituting the sample autocorrelation coefficients $\hat{\rho_{1}} \ldots \hat{\rho_{p}}$ into the first $p$ Yule-Walker equations and solving for $\left(\hat{\alpha}_{1}, \ldots \hat{\alpha}_{p}\right)$ (for further details see [4]). In matrix form these equations are :-

$$
\mathbf{R} \hat{\alpha}=\mathbf{r}
$$

where

$$
\mathbf{R}=\left(\begin{array}{ccccc}
1 & \rho_{1} & \rho_{2} & \ldots & \rho_{p-1} \\
\rho_{1} & 1 & \rho_{2} & \ldots & \rho_{p-2} \\
\rho_{2} & \rho_{3} & 1 & \ldots & \rho_{p-3} \\
\ldots & \ldots & \ldots & \ldots & \ldots \\
\rho_{p-1} & \rho_{p-2} & \rho_{p-3} & \ldots & 1
\end{array}\right) \hat{\alpha}=\left(\begin{array}{c}
\hat{\alpha}_{1} \\
\hat{\alpha}_{2} \\
\vdots \\
\hat{\alpha}_{p-1} \\
\hat{\alpha}_{p}
\end{array}\right) \mathbf{r}=\left(\begin{array}{c}
\rho_{1} \\
\rho_{2} \\
\vdots \\
\rho_{p-1} \\
\rho_{p}
\end{array}\right)
$$

The order $p$ is estimated using the partial autocorrelation function and the Akaike's Information Criterion (AIC) [1].

\subsubsection{The Partial Autocorrelation Function}

When fitting an $\operatorname{AR}(p)$ model, the last coefficient, $\alpha(p)$ measures the excess correlation at lag $p$ not captured by an $\operatorname{AR}(p-1)$ model. The partial autocorrelation function (pcf) is a function of the order, $\mathrm{p}$ and the last coefficient, $\alpha(p)$. By fitting AR processes of successively higher order $m$ and taking the estimate for $\hat{\alpha}_{m}$, to be approximately normally distributed, we can test the null hypothesis that $\alpha_{m}=0$ and hence that the order of the AR process is $m-1$. It can be shown that $\hat{\alpha}_{m}$ lies in the band $\pm 2 / \sqrt{N}$ with probability $95 \%$ if $\alpha_{m}=0$, assuming a Normal distribution [23].

The pcfs of the aggregate and web data from data set $\mathrm{t} 9702061439$ are shown in Figure 9. It can be seen that in this instance, the order is not obvious, and we thus use the AIC. 

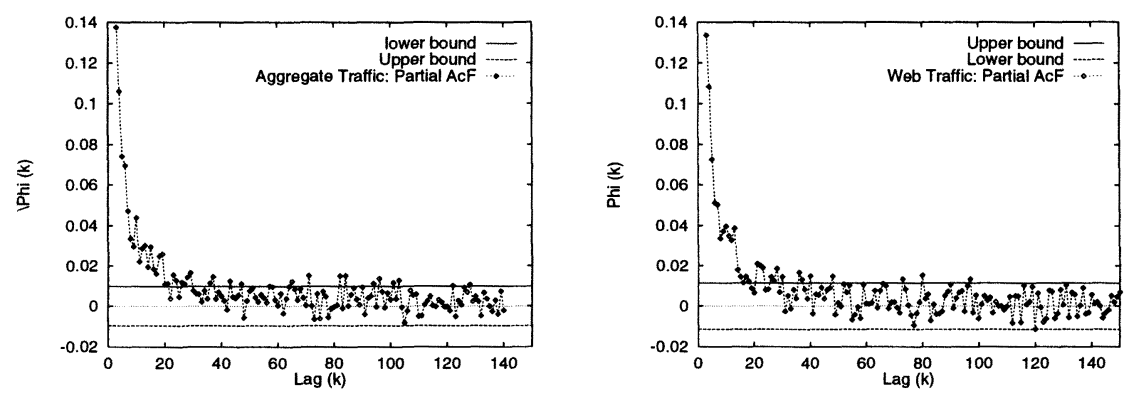

Figure 9: Partial autocorrelation function of inter-packet times. Ref : Tt9702061439

\subsubsection{The Akaike's Information Criterion (AIC)}

This is a very general criterion based on information theoretic concepts. It uses the quantity:

$$
\begin{aligned}
\operatorname{AIC}(q) & =n \log \hat{\hat{\sigma_{\epsilon}^{2}}}+2 q \text { where } \\
\hat{\hat{\sigma_{\epsilon}^{2}}} & =\hat{\phi}(0)+\hat{\alpha}_{1} \hat{\phi}(1)+\ldots+\hat{\alpha}_{1} \hat{\phi}(k)
\end{aligned}
$$

$\hat{\phi}(k)$ is the estimated covariance at lag $\mathrm{k}$. If we plot AIC(q) against $\mathrm{q}$, the graph in general will show a clear minimum and the appropriate order of the model is determined by the value of $q$ at which $\operatorname{AIC}(q)$ attains its minimum value. The book by Priestley [22] as well as the original paper by Akaike [1] give full details on the workings of the AIC. In this case, the plot shows a minimum value at 40 which is therefore taken to be the order of the AR model.

\subsection{The MMPP model}

Though we have characterised the inter-arrival time distribution of ATM cells as a stochastic model, a time series model is inappropriate in analytical queueing network models. Hence we seek to show a link between the autocorrelation function of inter-arrival times from the sampled traffic and the well known Markov Modulated Poisson Process (MMPP). We then use a MMPP, which has been parameterised using the autocorrelation function, to model correlated ATM traffic.

The MMPP is a doubly stochastic Poisson process and was first used in queueing theory by Naor and Yelachi [17] and Neuts [18]. Poisson arrivals are generated by a source with rate governed by an $m$-state irreducible continuoustime Markov Chain (CTMC) which is independent of the instantaneous arrival process. 
While the underlying CTMC is in state or phase $i$, let arrivals occur according to a Poisson process with rate $\lambda_{i}$. The MMPP is characterised by the following parameters :-

1. The generator matrix of the underlying modulating CTMC, $Q$.

$$
Q=\left(\begin{array}{cccc}
-q_{1} & q_{12} & q_{13} & \ldots \\
q_{21} & -q_{2} & q_{23} & \ldots \\
\vdots & & & \vdots \\
q_{m 1} & & \ldots & -q_{m}
\end{array}\right)
$$

where $q_{i}=\sum_{j=1 j \neq i}^{m} q_{i j}$ and $q_{i j}$ is the instantaneous transition rate from $i$ to $j$. In the sequel, the transition rate matrix $Q$ is assumed to be timehomogeneous, i.e. the process we are modelling is stationary and therefore $Q$ does not vary with time.

2. The Poisson arrival rate at each phase is given by the diagonal matrix,

$$
\Lambda=\left(\begin{array}{cccc}
\lambda_{1} & 0 & \ldots & 0 \\
0 & \lambda_{2} & \ldots & 0 \\
\vdots & & & 0 \\
0 & 0 & \ldots & \lambda_{m}
\end{array}\right)
$$

\subsubsection{Joint inter-arrival time distribution}

In order to find the inter-arrival time distribution of an MMPP, we use the sequence $\left\{\left(J_{k}, X_{k}\right), k \geq 0\right\}$ where $X_{k}$ is the time between the $(k-1)$ th and $k$ th arrivals and $J_{k}$ is the state of the CTMC at the $k$ th arrival instant. See also [8]. The resulting Markov renewal sequence has joint state-transition/inter-arrival time probability distribution matrix given by

$$
\begin{aligned}
F(x) & =\int_{0}^{x} e^{[(Q-\Lambda) u]} d u \Lambda \\
& =\left[-e^{(Q-\Lambda) u}(\Lambda-Q)\right]_{0}^{x} \Lambda \\
& =\left\{\mathbf{I}-e^{(Q-\Lambda) x}(\Lambda-Q)^{-1} \Lambda\right.
\end{aligned}
$$

Each of the elements of $F_{i j}(x)$ are the conditional probabilities, $P\left\{J_{k}=j, X_{k} \leq\right.$ $\left.x \mid J_{k-1}=i\right\}$ for $k \geq 1$. The corresponding probability density function is

$$
\begin{aligned}
f(x) & =\frac{d}{d x} F(x)=\left\{e^{-(\Lambda-Q) x}(\Lambda-Q)\right\}(\Lambda-Q)^{-1} \Lambda \\
& =e^{(Q-\Lambda) x} \Lambda
\end{aligned}
$$

The joint density of $X_{1}, \ldots X_{n}$, with the state $j_{n}(n \geq 1)$, conditioned on the state $j_{0}$, is the $n$-fold convolution of the probability density function matrix. Its joint Laplace transform is therefore:

$$
f^{*}\left(s_{1}, \ldots, s_{n}\right)=\prod_{k=1}^{n}\left(s_{k} \mathbf{I}-Q+\Lambda\right)^{-1} \Lambda
$$




\subsubsection{The autocorrelation function of the inter-arrival time distri- bution}

We determine the conditional moments of the time between arrivals by taking partial derivatives of the Laplace transform of the joint probability density function matrix for a sequence of $n$ successive arrival instants. We then decondition over the initial states (assuming stationarity) and sum over the final states. We can then obtain an MMPP in terms of the autocovariance function of the underlying time series.

Let $\pi$ represent the stationary vector of $Q$ (eq. 4) of the CTMC that satisfies $\pi Q=0$ and $\sum_{i=1}^{m} \pi_{i}=1$ and let $\mathbf{e}=(1, \ldots, 1)^{T}$. Let the equilibrium probability that an arbitrary arrival finds the CTMC in state $i$ be $\pi_{i}^{*}$. Then,

$$
\pi_{i}^{*}=\frac{\pi_{i} \lambda_{i}}{\sum_{j=1}^{m} \pi_{j} \lambda_{j}}
$$

i.e. the state probability vector on arrival is :-

$$
\pi^{*}=\frac{\pi \Lambda}{\pi \cdot \lambda}
$$

Thus

$$
E\left[X_{n}^{l}\right]=\pi^{*} l !\left[(\Lambda-Q)^{-1} \Lambda\right]^{n-1}(\Lambda-Q)^{-(l+1)} \Lambda \mathbf{e}, \quad l \geq 1
$$

In particular, the first moment of $X_{k}$ is given by :-

$$
E\left[X_{k}\right]=\pi^{*}\left[(\Lambda-Q)^{-1} \Lambda\right]^{k-1}(\Lambda-Q)^{-2} \Lambda \mathbf{e}, \quad 1 \leq k \leq n
$$

The expected value of the product $X_{1} X_{k+1}$ is given similarly by taking partial derivatives of $f^{*}\left(s_{1}, \ldots s_{k+1}\right)$ w.r.t. $s_{1}$ and $s_{k+1}$ at $s_{1}=s_{2} \ldots=s_{k+1}=0$.

$$
E\left[X_{1} X_{k+1}\right]=\pi^{*}(\Lambda-Q)^{-2} \Lambda\left[(\Lambda-Q)^{-1} \Lambda\right]^{k-1}(\Lambda-Q)^{-2} \Lambda \mathbf{e}
$$

The autocovariance at lag $\mathrm{k}, E\left[\left(X_{1}-E\left[X_{1}\right]\right)\left(X_{k+1}-E\left[X_{k+1}\right]\right)\right]=E\left[X_{1} X_{k+1}\right]-$ $E\left[X_{1}\right] E\left[X_{k+1}\right]$, is therefore,

$$
\phi_{k}=\pi^{*}(\Lambda-Q)^{-2} \Lambda\left[\left\{\mathbf{I}-\mathbf{e} \pi^{*}(\Lambda-Q)^{-1} \Lambda\right\}\left\{(\Lambda-Q)^{-1} \Lambda\right\}^{k-1}\right](\Lambda-Q)^{-2} \Lambda \mathbf{e}, k \geq 1
$$

The autocovariance at lag 0 i.e. the variance of inter-arrival times is given by :-

$$
\begin{aligned}
\phi_{0} & =E\left[X_{1}^{2}\right]-E\left[X_{1}\right]^{2} \\
& =2 \pi^{*}(\Lambda-Q)^{-3} \Lambda \mathbf{e}-\left(\pi^{*}(\Lambda-Q)^{-2} \Lambda \mathbf{e}\right)^{2}
\end{aligned}
$$

The autocorrelation function for a MMPP, $\rho_{k}=\phi_{k} / \phi_{0}$, for $k \geq 1$ is thus :-

$$
\frac{\pi^{*}(\Lambda-Q)^{-2} \Lambda\left[\left\{\mathbf{I}-\mathbf{e} \pi^{*}(\Lambda-Q)^{-1} \Lambda\right\}\left\{(\Lambda-Q)^{-1} \Lambda\right\}^{k-1}\right](\Lambda-Q)^{-2} \Lambda \mathbf{e}}{2 \pi^{*}(\Lambda-Q)^{-3} \Lambda \mathbf{e}-\left(\pi^{*}(\Lambda-Q)^{-2} \Lambda \mathbf{e}\right)^{2}}
$$




\subsubsection{Mean of the Inter-arrival times (IAT)}

The first moment (mean) of the IAT is (from Eq. 6) :-

$$
\begin{aligned}
E\left[X_{1}\right] & =\pi^{*}(\Lambda-Q)^{-2} \Lambda \mathbf{e} \\
& =\frac{\pi \Lambda(\Lambda-Q)^{-1}(\Lambda-Q)^{-1} \Lambda e}{\pi \cdot \lambda} \\
& =\frac{\pi\left(\mathbf{I}+Q(\Lambda-q)^{-1}\right)\left(\mathbf{I}+(\Lambda-q)^{-1} Q\right) \mathbf{e}}{\pi \lambda} \\
& =\frac{1}{\pi \lambda}
\end{aligned}
$$

as $\pi \cdot Q=0$ and $Q . e=0$, as expected.

\subsection{Parameterising a MMPP}

We present a method to parameterise a MMPP by matching the first moment and the observed autocorrelations against those of the MMPP given by eq. 7 in section 4.2.2. If $(n-1)$ covariances are to be taken into account, we would need at least $n$ equations to parameterise the MMPP in this way, which would then have order of at least Ceil $[\sqrt{n}]$. Eqn 8 and Eqn. 7 are used to match the mean and autocorrelations respectively.

The parameters are found numerically by solving a set of non-linear equations; we use Broyden's method - a multidimensional secant method.

To validate our approach, we first estimate the parameters of a MMPP from the mean IAT and autocorrelation function of a known MMPP, with generator matrix $\left\{q_{i, j}\right\}$ and rate-matrix $\left\{\lambda_{i}\right\}$. Preliminary results, shown in Table 2 indicate that the method does not converge to a unique solution for different initial values to the numerical method. The autocorrelations obtained from each solution, as expected, are very close to the autocorrelations of the original MMPP. This is not entirely surprising since, although a given stochastic process has a unique covariance structure, the converse is not true. It is usually possible to find many normal and non-normal processes with the same acf. Jenkins and Watts [13] (p. 170) give an example of two different stochastic processes which have the same acf. Since our objective is to match autocorrelations, any such process is acceptable. Other criteria might prefer one of the possible candidates, but we do not consider this further here. The algorithm suffers from the limitations of Broyden's method, that the initial values must be close to the roots of the equation for non-linear systems. More importantly, if the Jacobian used in the calculation of the next estimates of the roots of the equations becomes singular or nearly singular, then the difference between the current estimate of the root and the next estimate cannot be determined.

\subsection{Known Autoregressive models}

When it is known that traffic of a certain type or from a particular channel has a certain stationary AR model (after subtracting out the mean), its autocor- 


\begin{tabular}{|c|c|c|c|}
\hline Parameter & True solution & Set 1 & Set 2 \\
\hline$q_{1,1}$ & -0.5 & -8.660272 & -0.454088 \\
$q_{1,2}$ & 0.5 & 8.660272 & 0.454088 \\
$q_{2,1}$ & 0.2 & 0.240278 & 0.198401 \\
$q_{2,2}$ & -0.2 & -0.240278 & -0.198401 \\
$\lambda_{1}$ & 0.9 & 8.009269 & 0.856416 \\
$\lambda_{2}$ & 0.1 & 0.115861 & 0.097955 \\
\hline
\end{tabular}

Table 2: Parameters for the MMPP

\begin{tabular}{|c|l|l|l|}
\hline Lag & Autocovariance & Autocovariance & Autocovariance \\
\hline & True Solution & Set 1 & Set 2 \\
\hline Mean & 3.04348 & 3.03997 & 3.04341 \\
1 & 0.063202247191 & 0.0552495292 & 0.063195764 \\
2 & 0.017775632022 & 0.0132967083 & 0.017773341687 \\
3 & 0.004999396506 & 0.0032000716 & 0.00499862097 \\
4 & 0.0014060802674 & 0.000770149 & 0.0014058252 \\
5 & 0.000395460075 & 0.000185342 & 0.00039537794 \\
6 & 0.000111223146 & 0.000044602 & 0.00011119712 \\
7 & 0.0000312815098 & 0.0000107351 & 0.00003127336 \\
8 & 0.0000008797924 & 0.000000258 & 0.000000879540399 \\
9 & 0.0000002474416 & 0.0000000621803 & 0.000000247364 \\
10 & $6.959295 * 10^{-} 7$ & $1.496472 * 10^{-} 7$ & $6.9569362950 * 10^{-} 7$ \\
\hline
\end{tabular}

Table 3: The acf for the first 10 lags 
relation function can be obtained analytically and the method of the previous subsection applied to obtain the corresponding MMPP directly. (An autoregressive process, $X_{t}=\alpha_{1} X_{t-1}+\alpha_{2} X_{t-2}+\ldots+\alpha_{k} X_{t-k}+Z_{t}$ is stationary if and only if the roots of the equation $1-\alpha_{1} B-\alpha_{2} B^{2} \ldots-\alpha_{3} B^{k}=0$ lie outside the unit circle.)

Consider now an $\mathrm{AR}(2)$ process

$$
X_{t}=\alpha_{1} X_{t-1}+\alpha_{2} X_{t-2}+Z_{t}
$$

Multiplying by $X_{t-k}$ and taking expectations, we obtain

$$
\phi_{k}=\alpha_{1} \phi_{k-1}+\alpha_{2} \phi_{k-2}+E\left[X_{t-k} Z_{t}\right]
$$

for the covariance $\phi_{k}$ between $X_{t}$ and $X_{t-k}$. Now, $E\left[X_{t-k} Z_{t}\right]=0$, when $k>0$ since $X_{t-k}$ can only involve shocks $Z_{t}$ up to time $t-k$ (see [3]). Thus

$$
\phi_{k}=\alpha_{k-1} \phi_{k-1}+\alpha_{2} \phi_{k-2} \text { for } k>0 .
$$

Similarly,

$$
\begin{aligned}
& \phi_{0}=\alpha_{1} \phi_{1}+\alpha_{2} \phi_{2}+\sigma_{Z}^{2} \quad \text { and for } k=1,2 \text { we have } \\
& \phi_{1}=\alpha_{1} \phi_{0}+\alpha_{2} \phi_{1} \\
& \phi_{2}=\alpha_{1} \phi_{1}+\alpha_{2} \phi_{0}
\end{aligned}
$$

Hence,

$$
\begin{aligned}
& \phi_{0}=\frac{\sigma_{Z}^{2}\left(1-\alpha_{2}\right)}{1-\alpha_{2}^{3}-\alpha_{2}^{2}-\alpha_{1}^{2}-\alpha_{1}^{2} \alpha_{2}-\alpha_{2}} \\
& \phi_{1}=\frac{\sigma_{Z}^{2} \alpha_{1}}{1-\alpha_{2}^{3}-\alpha_{2}^{2}-\alpha_{1}^{2}-\alpha_{1}^{2} \alpha_{2}-\alpha_{2}}
\end{aligned}
$$

\subsubsection{An example}

Consider the following second-order stationary $\mathrm{AR}(2)$ process:

$$
X_{t}=\frac{1}{3} X_{t-1}+\frac{2}{9} X_{t-2}+Z_{t}
$$

We calculate the autocorrelation function of the $\mathrm{AR}(2)$ model using the equations in section 4.4 and then use our numerical routines to obtain an MMPP that has the same autocorrelation function for the first 4 lags (recall that the mean has been subtracted out). The parameters of the best-fit MMPP turn out to be

$$
\begin{aligned}
& Q=\left(\begin{array}{cc}
-0.015004 & 0.015004 \\
0.108 & -0.108
\end{array}\right) \\
& \Lambda=\left(\begin{array}{cc}
10.0 & 0 \\
0 & 0.4
\end{array}\right)
\end{aligned}
$$




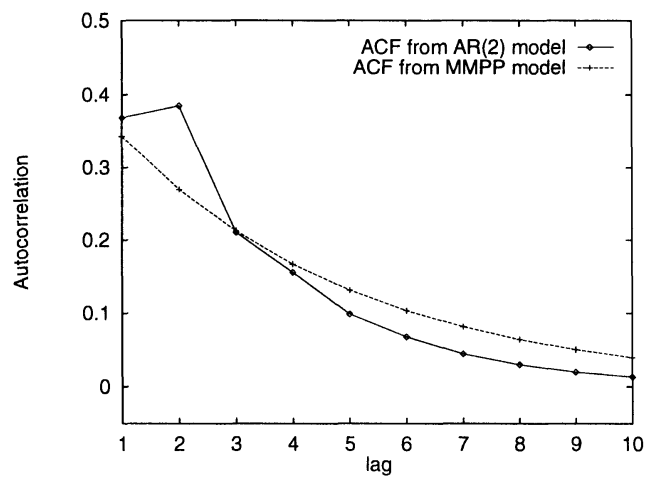

Figure 10: A comparision of the autocorrelation functions

We then plot the first 10 autocorrelations of both the AR process and the autocorrelations from a simulation of the derived MMPP model. Results are shown in fig. 10.

The AIC for the time series resulting from the MMPP was also calculated and is plotted in Fig. 11. Clearly the order is 2 indicating that the order of the original time series has been preserved.

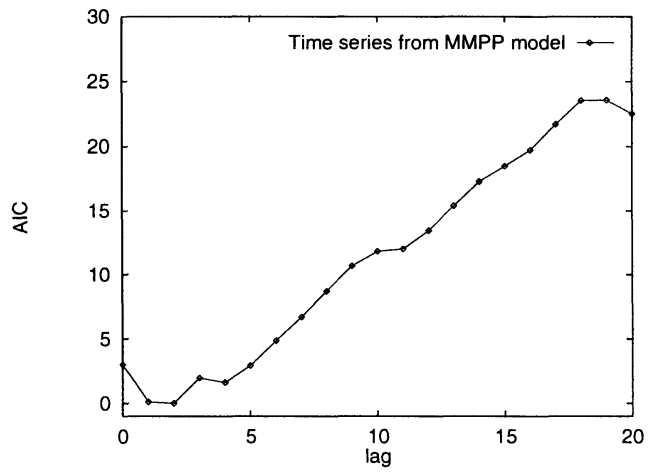

Figure 11: AIC from analytical autocorrelation function

\subsection{MMPP for traffic data}

To fit an MMPP against real, monitored traffic data, we applied the method to the e-mail traffic from observed data set Tt9702061439. We first estimate the autocorrelation function of the time series comprising the observed arrival 
instants, $\left\{A_{i} \mid 1 \leq i \leq n\right\}$. At lag $k(0 \leq k<n)$. It is estimated as

$$
\rho_{k}=\frac{\phi_{k}}{\phi_{0}}, \text { where } \hat{\phi}_{k}=\frac{1}{n-k-1} \sum_{i=1}^{n-k-1} X_{i+k} X_{i}
$$

where $X_{i}=\Delta_{i}-\bar{\Delta}(1 \leq i \leq n-1), \Delta_{i}=A_{i+1}-A_{i}$ and $\bar{\Delta}=E\left[\Delta_{i}\right]$.

From these estimates, we match a 2-phase MMPP as in the previous sections, using the mean, $\hat{\mu}_{1}, \hat{\rho}_{1}, \hat{\rho}_{2}$ and $\hat{\rho}_{3}$ to determine the parameters of the MMPP and use autocorrelations at higher lags for validation. The MMPP that we estimate is :-

$$
\begin{aligned}
& Q=\left(\begin{array}{cc}
-1.1 & 1.1 \\
0.7 & -0.7
\end{array}\right) \\
& \Lambda=\left(\begin{array}{cc}
9.0 & 0 \\
0 & 2.0
\end{array}\right)
\end{aligned}
$$

Mean for the traffic data : 0.213761 ; Mean for MMPP model : 0.211765 .

\section{The burst size distribution}

The fit in Fig. 6 led us to the conclusion that there is burstiness at 2 distinct levels; at the primary level, due to batch arrivals of cells within a packet with inter-arrival time of order $200-1000 \mathrm{~ns}$ and at a secondary level due to the effect of TCP and is of order $2000-7000 \mathrm{~ns}$.

\subsection{Cell level}

To examine the burst size distribution at the ATM cell level, we plotted histograms for the numbers of cell arrivals within a packet. The distribution for heavy traffic is shown in Figure 12 and that for light traffic sites in Fig. 13.

It is evident from the histograms that both heavy utilisation channels and low-utilisation channels have a similar burst size distribution. This can be explained by the type of traffic that is flowing in the network. Most of the traffic is TCP/IP traffic that has originated from an Ethernet LAN. The MAN has been configured with AAL5 which is the data transport layer of ATM and is used to send IP datagrams. When sending these, IP does not fragment to the ATM cell size [5],[6],[9] (48 octets +5 octets header information) itself: instead, it uses a Maximum Transport Unit (MTU) of 9180 octets and allows AAL5 to fragment the datagram to cells. At the LAN level, Ethernet imposes a MTU of 1518 octets and a minimum of 64 octets. From this it is evident that the minimum number of cells that will be sent is Ceil[64/48] $=2$ and the maximum in one batch $=$ Ceil[1518/48] $=32$. In the histograms showing the frequency of burst-size distributions, three bin sizes, 2, 12 and 32 were found to occur 

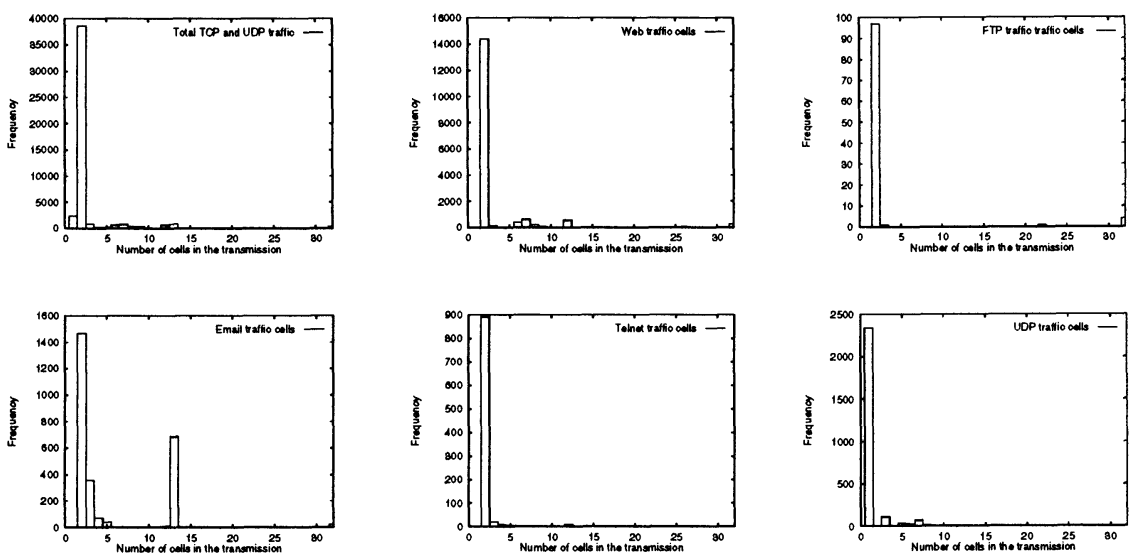

Figure 12: Burst size distributions Set : t9702041420
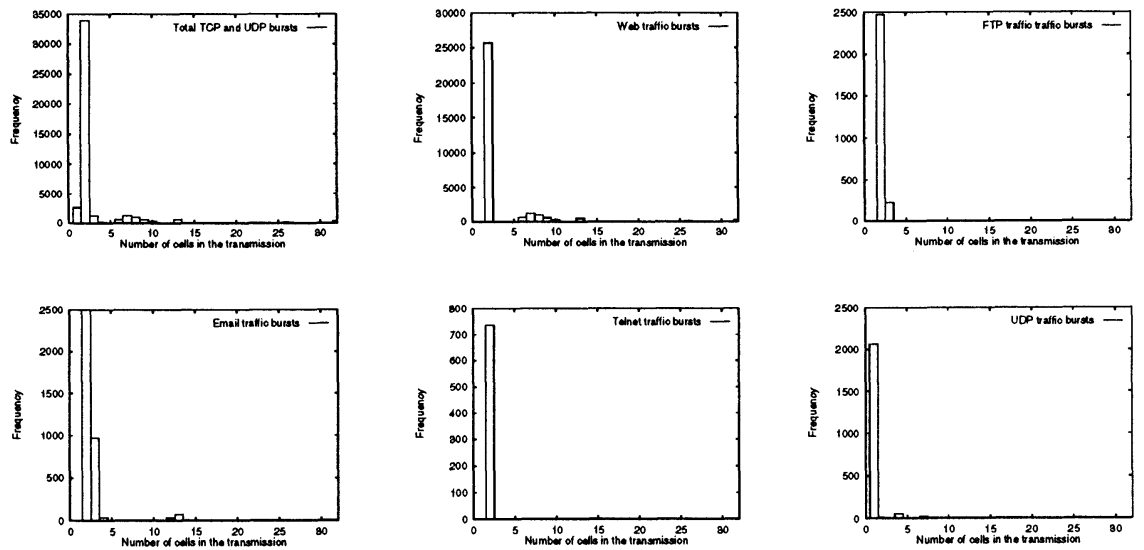

Figure 13: Burst size distributions Set : t9702061439 


\begin{tabular}{|c|l|l|c|c|}
\hline Figure & $\chi^{2}$ statistic & $\nu$ & $\chi_{0.025, \nu}^{2}$ & Accept? \\
\hline A & 787.000 & 15 & 30.58 & No \\
B & 21851.800 & 15 & 30.58 & No \\
C & 259.000 & 15 & 30.58 & No \\
D & 17.168 & 8 & 17.53 & Yes \\
\hline
\end{tabular}

Table 4: Goodness of Fit

invariably in histograms for aggregate traffic, Web traffic and FTP traffic. 2 is by far the most frequent due to the acknowlegement packets that are involved in TCP/IP transfers. Web and FTP packets tend to be large and are the largest constituents of the traffic. There is thus likely to be fragmentation in the transportation of their packets. The peak at bin size 12 corresponds to the IP specification that says that a router must always handle datagrams of up to 576 octets. Ceil $[576 / 48]=12$.

\subsection{TCP Level}

The geometric distribution has been used widely to model burstiness in network traffic [10],[21],[14]. We investigate whether it is suitable for modelling the TCP batch size distribution from our traffic data. We fitted an exponential function, $a e^{-\lambda x}$; see Figure 14. A geometric random variable has probability mass function $p_{i}=p(1-p)^{i-1}$ for $i \geq 1$ and some positive $p<1$. Comparing with the fitted exponential function, we have

$$
1-p=e^{-\lambda}
$$

To show the fit clearly, we have drawn the histograms using a line plot rather than a normal box plot by joining the mid-points of each bar of the histogram. To test the goodness of fit, we performed the $\chi^{2}$ test on the fits and obtained the results shown in Table 4 . Thus at the $97 \%$ significance level, we conclude that the geometric distribution is not suitable for modelling TCP batch size distributions in Figs. 14A - 14C, but seems to be suitable in the case of Fig. 14D. The reason that Figs. 14A, 14B and 14C show such a bad fit is the small values in the tails of the fitted exponential function. However, the test on $14 \mathrm{D}$ does show that the geometric distribution may be a good model for ATM e-mail batch sizes. The use of generalised exponential distribution (GE) would then model an e-mail arrival process in heavy traffic well. However a more general model would have to model more closely the MTUs of different networks and thus the fragmentation that occurs as data travels through them.

\section{Conclusions}

The results presented in this paper show that ATM traffic shows very different behaviour depending on its intensity. This has a direct bearing on modelling input traffic in analytical models. 
A

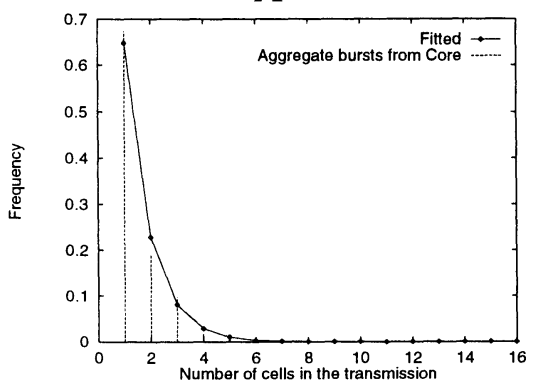

C

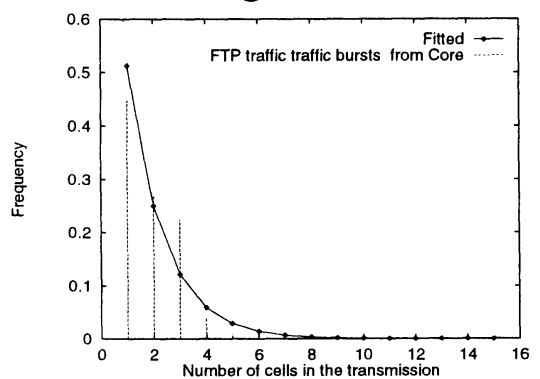

B

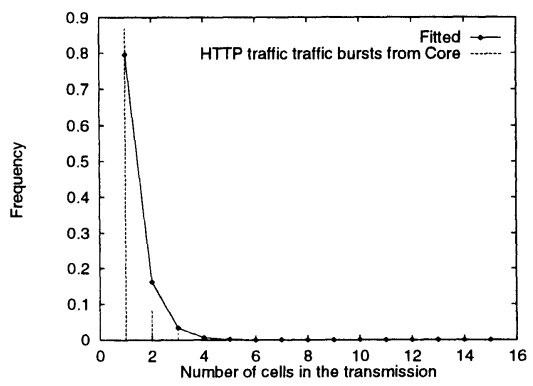

D

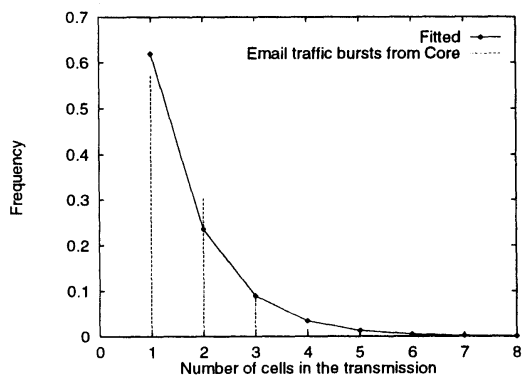

Figure 14: Burst size distribution Set : t9702041154 
We have shown that heavy traffic on the MAN can be accurately modelled by a Poisson point process with 2 levels of burstiness. We have shown how the sizes of the bursts are a consequence of fragmentation within the networks and from the results in section 5.2, it is evident that a geometric distribution can be a good model for the burst size distribution in some cases. Though we suspect that a deterministic model based on the workings of TCP may be better, such models are not mathematically tractable in analytical modelling and queueing network models in particular. Moreover, experience suggests that many model outputs are sensitive to only mean burst size and that the assumption of geometrically distributed burst sizes is robust.

Light traffic exhibits long-memory or long range correlation. We have proposed a method to model it with a MMPP parameterised using the autocorrelations of the time series obtained from our data. The MMPP, of course, has the added advantage of being analytically tractable. The MMPP has been suggested as a model for ATM traffic, see [11], [25],[19] and a fitting method based on the EM algorithm [7] has been used to parameterise it in [16]. However, our approach is novel as we use correlation rather than the burstiness in the traffic to parameterise our MMPP model. It is our hope that this coupled with spectral expansion techniques will lead to building blocks for queueing network models for bursty, correlated arrivals.

In time series analysis, periodic effects frequently emerge strongly in long time spans and it is a common method to use self-similarity in the study of such time series. It is therefore likely that in previous publications where mention of self-similarity and fractal-like behaviour has been made, a simple explanation of the observed behaviour may be that of periodicity which could be due to the TCP/IP protocols or usage patterns. Also many of the traffic measurements used in e.g. [20] and [26] were done prior to the advent of the web and therefore exclude the massive increase in the volume of traffic due to it.

Finally, we have also proposed a new method to parameterise an MMPP. Based on our preliminary investigations the results look encouraging. A general problem is that it is not at all clear that an arbitrary autocovariance function can be realised by some MMPP. There is little that can be done about this but a possible approach to overcoming the it is the following. An observed time series could first be modelled by an ARMA process using established techniques for which software already exists, e.g. SPlus. This step is intended to characterise the process generally, rather than represent one particular sample which is prone to producing significant estimation errors. If successful, the autocorrelation function of the ARMA process would provide a more reliable representation of the real traffic and the method of section 4.4 could be used.

\section{References}

[1] $\mathrm{H}$ Akaike. A new look at the statistical model identification. IEEE Trans Auto. Control, 19:716 - 723, 1974. 
[2] M D K Bhabuta. Quantitative analysis of ATM networks. PhD thesis, Imperial College of Science, Tech. and Med., 1998.

[3] Box and Jenkins. Time Series Analysis. Holen-Day, 1970.

[4] C Chatfield. The analysis of time series, An Introduction. Chapman and Hall, 1996.

[5] D Comer. Internetworking with TCP/IP Vol I. Prentice-Hall, 1995.

[6] D Comer and D Stevens. Internetworking with TCP/IP Vol II. PrenticeHall, 1994.

[7] A Dempster, N Laird, and Rubin D. Maximum liklihood from incomplete data via the EM algorithm. J. of Roy. Statistical Soc. B, 39:1-38, 1977.

[8] W Fischer and K Meier-Hellstern. The markov-modulated poisson process (mmpp) cookbook. Performance Evaluation, 18:149-171, 1992.

[9] D Ginsburg. ATM, Solutions for enterprise networking. Addison-Wesley, 1996.

[10] P Harrison and A de C Pinto. An approximate analysis of asynchronous, packet-switched buffered banyan networks with blocking. Performance Evaluation, 19:223-258, 1994.

[11] H Heffes and D Lucantoni. A Markov modulated characterisation of packetized voice and data traffic and related statistical multiplexer performance. IEEE J. Selected Areas Comm., 4(6):856-868, 1986.

[12] Jain. The Art of Computer Systems Performance Analysis. John Wiley and Sons, 1991.

[13] Jenkins and Watts. Spectral Analysis and its applications. Holden-Day, 1968.

[14] D Kouvatsos, J Wilkinson, P Harrison, and M Bhabuta. Performance analysis of buffered MIN ATM switches. In ATM NETWORKS, Performance Modelling and Analysis, 1996.

[15] S Manthrope, I Norros, and J Le Boudec. The second-order characteristics of TCP. ACM/Sigmetrics, 1996.

[16] K Meier-Hellstern. Fitting algorithm for MMPP having two arrival rates. European Journal of Operations Research, 29:370-377, 1987.

[17] P Naor and U Yechiali. Queueing problems with hetrogenous arrivals and service. Operations Research, 19:722-734, 1971.

[18] M Neuts. A versatile Markovian point process. J. of Appl. Probability, 16:764-779, 1979. 
[19] B Nielsen. Modelling of multiple access systems with phase type distributions. PhD thesis, The Technical University of Denmark, 1988.

[20] V Paxon and S Floyd. Wide area traffic: The failure of Poisson modeling. IEEE/ACM Transactions on Networking, 3(3):226-245, June, 1995.

[21] A de C Pinto. Models of finite-buffered packet-switched multistage interconnection networks. PhD thesis, Imperial College of Science, Tech. and Med., 1994.

[22] M Priestley. Spectral Analysis and Time Series. Academic Press, 1989.

[23] M Quenouille. Approximate tests of correlation in time series. J. Roy. Satist. Soc., Ser B(11):68-84, 1949.

[24] B Ray. Modeling long-memory processes for optimal long-range prediction. J. of Time Series Analysis, 14(5):511-525, 1993.

[25] K Sriram and W Whitt. Characterizing superposition arrival proceses in packet multiplexers for voice and data. IEEE J. Selected Areas Comm., 4(6):833-846, 1986.

[26] M Taqqu, W Wallinger, and W Wilson. On the self-similar nature of Ethernet traffic. IEEE/ACM Transactions on networking, 2(1):1-13, February, 1994. 